NOTE

\title{
Vertical profiles of Iodine-131 and Cesium-137 in soils in Fukushima Prefecture related to the Fukushima Daiichi Nuclear Power Station Accident
}

\author{
Kazuya Tanaka, ${ }^{1 *}$ Yoshio Takahashi, ${ }^{2}$ Aya SaKaguchi,${ }^{2}$ Miyuki Umeo, ${ }^{2}$ Shinjiro Hayakawa, ${ }^{3}$ \\ HaJime TANida, ${ }^{4}$ TAKashi SAITO $^{5}$ and YUtAKa KanAi ${ }^{6}$ \\ ${ }^{1}$ Institute for Sustainable Sciences and Development, Hiroshima University, \\ 1-3-1 Kagamiyama, Higashi-Hiroshima, Hiroshima 739-8530, Japan \\ ${ }^{2}$ Graduate School of Science, Hiroshima University, 1-3-1 Kagamiyama, Higashi-Hiroshima, Hiroshima 739-8526, Japan \\ ${ }^{3}$ Graduate School of Engineering, Hiroshima University, 1-4-1 Kagamiyama, Higashi-Hiroshima, Hiroshima 739-8527, Japan \\ ${ }^{4}$ Office of Society-Academia Collaboration for Innovation, Kyoto University, \\ Yoshida-Honmachi, Sakyo-ku, Kyoto 606-8501, Japan \\ ${ }^{5}$ Fukushima Agricultural Technology Centre, Hiwada, Koriyama, Fukushima 963-0531, Japan \\ ${ }^{6}$ Geological Survey of Japan, National Institute of Advanced Industrial Science and Technology (AIST), \\ 1-1-1 Higashi, Tsukuba, Ibaraki 305-8567, Japan
}

(Received August 4, 2011; Accepted October 11, 2011)

\begin{abstract}
Vertical profiles of iodine-131 and cesium-137 emitted from the Fukushima Daiichi Nuclear Power Station were determined in soil core samples collected in Fukushima prefecture. The profiles showed that more than $76 \%$ of the radionuclides were retained within $5 \mathrm{~cm}$ of the surface. Leaching experiments showed that cesium was strongly bound to soil particles which could not be leached with $2 \mathrm{M} \mathrm{HCl}$, while more than $40 \%$ of iodine was incorporated into humic substances.
\end{abstract}

Keywords: Iodine-131, Cesium-137, clay minerals, humic substances, Fukushima

\section{INTRODUCTION}

An earthquake of magnitude 9.0 and a subsequent large tsunami that occurred on March 11, 2011 caused enormous damage to coastal cities in northeast Japan. In particular, the Fukushima Daiichi Nuclear Power Station was severely affected by the tsunami, which destroyed the cooling system of the station and induced several explosions from four reactors at the station (Butler, 2011). Consequently, a large amount of radionuclides was emitted into the surrounding environment. Among them, vertical profiles of iodine-131 (half life: 8.02 days) and cesium-137 (30.2 years) in soil core samples collected in Fukushima prefecture are reported in this note. Profiles are important information for the decontamination of the radionuclides from the soil, which are closely related to the radiation dose to people living in the area, transfer of the radionuclides to crops, and prediction of the migration of the radionuclides from the soil to other systems. Among radionuclides emitted in the accident, Imanaka $e t$

\footnotetext{
*Corresponding author (e-mail: kt0830@hiroshima-u.ac.jp)

Copyright @ 2012 by The Geochemical Society of Japan.
}

al. (2011) detected iodine-131 and cesium-137 in soil samples collected on March 29 in the prefecture using germanium semiconductor detectors. This study focuses on the vertical distributions of the two radionuclides in surface soil.

\section{SAMPLES AND METHODS}

Soil core samples were taken on April 13, 2011 at three sites in Fukushima prefecture: Hiwada in Koriyama-city

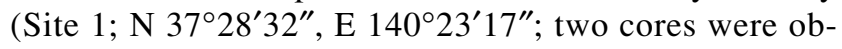
tained in Site 1), Yabuki in Nishi-Shirakawa-county (Site 2; N $37^{\circ} 11^{\prime} 59^{\prime \prime}$, E $140^{\circ} 20^{\prime} 37^{\prime \prime}$ ), and Iizaka in Fukushima-

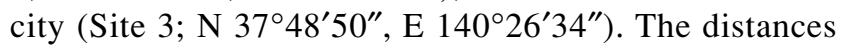
of the three sites from the Fukushima Daiichi Nuclear Power Station are $55 \mathrm{~km}$ (Site 1) and $65 \mathrm{~km}$ (Sites 2 and 3 ). We selected the three sites to investigate different types of soils. The soil types of the samples for Sites 1-3 were gray lowland soil, andosol, and brown forest soil, respectively. The soil at Site 1 was taken from a field, whereas the soil at Sites 2 and 3 was used to grow fruit trees. A stainless steel pipe with a diameter of $4.7 \mathrm{~cm}$ and length of $30 \mathrm{~cm}$ was inserted into the soil at each site to recover the soil core sample, which was divided into 8 fractions 

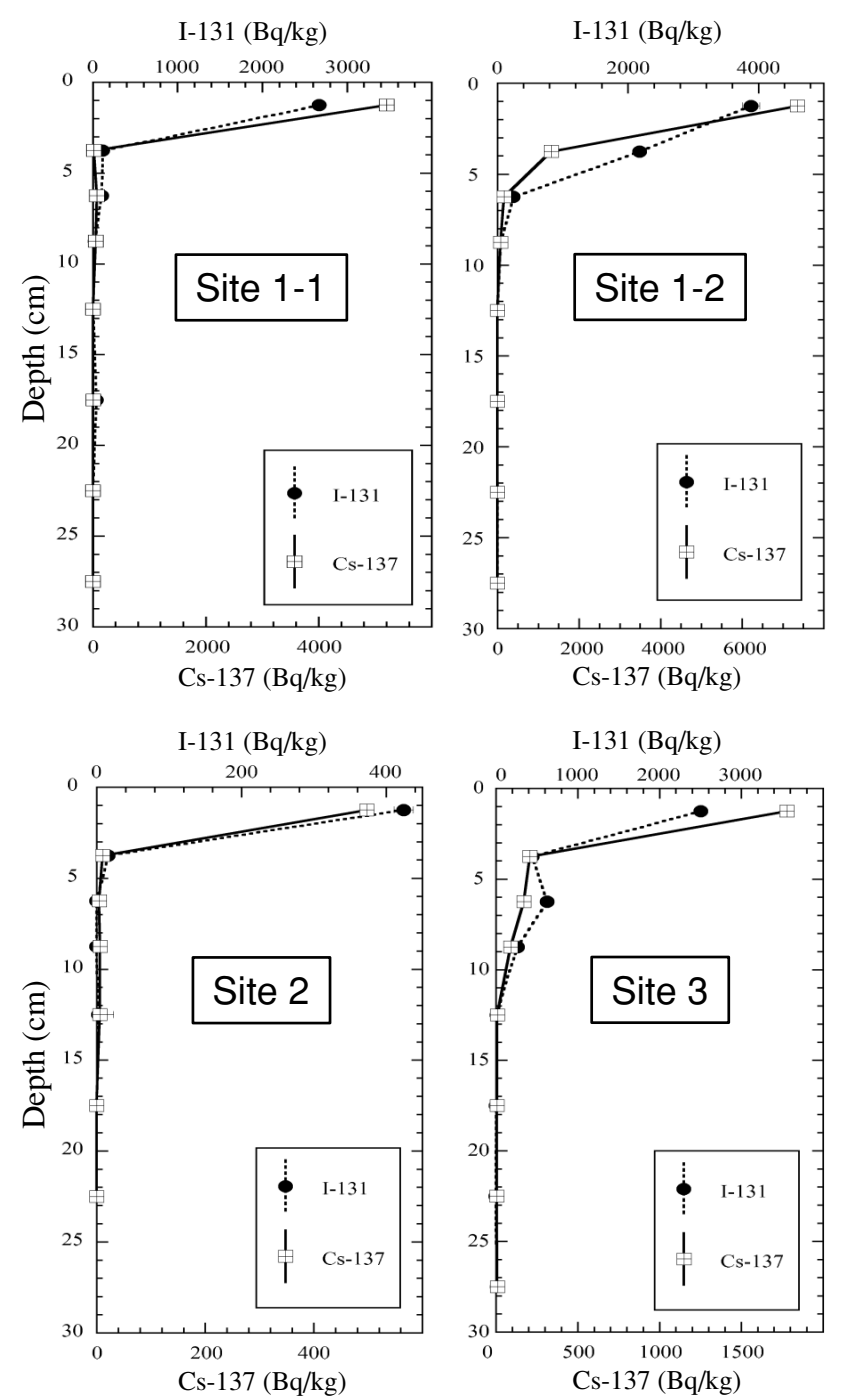

Fig. 1. Vertical profiles of iodine-131 and cesium-137 in the soil layer at Site 1 (two cores: Site 1-1 and Site 1-2), Site 2, and Site 3.

by depth: $0-2.5,2.5-5,5-7.5,7.5-10,10-15,15-20,20-$ 25 , and $25-30 \mathrm{~cm}$ from the surface. Each sample was sieved with a $2 \mathrm{~mm}$ mesh screen to remove pebbles and plant debris. The samples were not dried to prevent possible loss of iodine into gas phase due to the volatile nature of the element.

After homogenization, each sample was loaded into a cylindrical polystyrene container with inner diameter of $5.0 \mathrm{~cm}$, outer diameter of $5.6 \mathrm{~cm}$ and height of $6.8 \mathrm{~cm}$. The soil sample in the container was placed on a planar type germanium semiconductor detector (Seiko EG\&G) to determine the count rate of $\gamma$-rays emitted from iodine131 and cesium-137. The count rate was converted to their radioactivities based on the calibration using homemade standard materials (matrix: weathered granitic soil) con-
Table 1. Proportion of iodine-131 and cesium-137 contained in the soil layer from surface to 2.5 or $5.0 \mathrm{~cm}$ depth

\begin{tabular}{lccccc}
\hline Sample & \multicolumn{2}{c}{$(0-2.5 \mathrm{~cm}) /$ Total } & & \multicolumn{2}{c}{$(0-5.0 \mathrm{~cm}) /$ Total } \\
\cline { 2 - 3 } \cline { 5 - 6 } & $\begin{array}{c}\text { iodine-131 } \\
(\%)\end{array}$ & $\begin{array}{c}\text { cesium-137 } \\
(\%)\end{array}$ & & $\begin{array}{c}\text { iodine-131 } \\
(\%)\end{array}$ & $\begin{array}{c}\text { cesium-137 } \\
(\%)\end{array}$ \\
\hline Site 1-1 & 90.3 & 97.1 & & 94.1 & 97.5 \\
Site 1-2 & 61.0 & 82.4 & & 95.2 & 97.2 \\
Site 2 & 95.8 & 94.8 & & 99.4 & 96.8 \\
Site 3 & 64.7 & 78.1 & & 76.1 & 87.2 \\
\hline
\end{tabular}

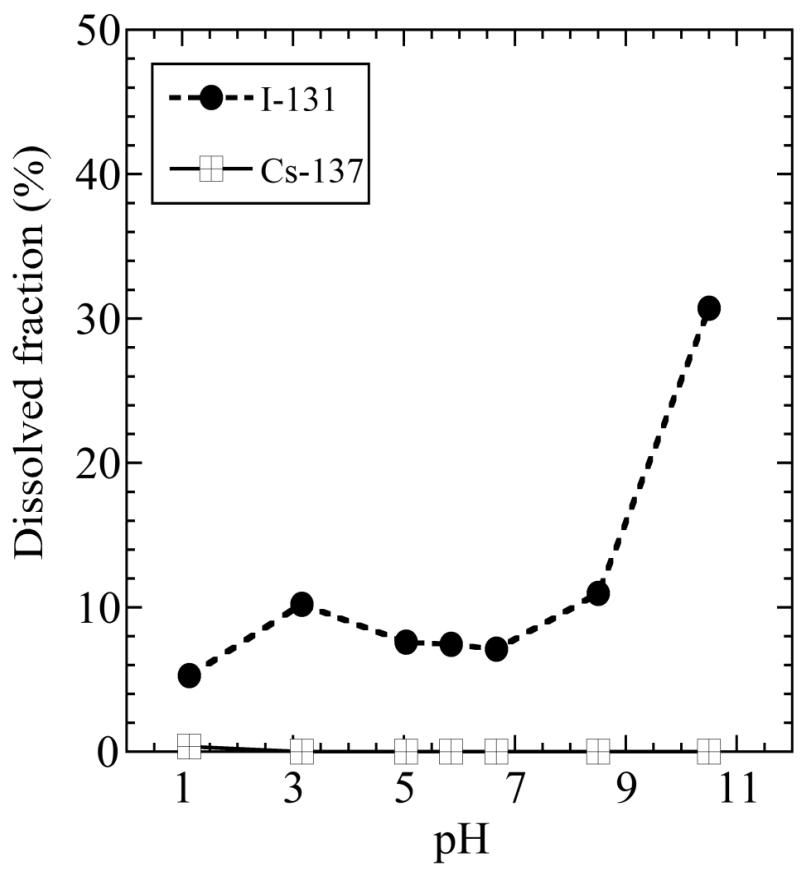

Fig. 2. Dissolved fraction of iodine-131 and cesium-137 from soil into water at various $\mathrm{pH}$.

taining known amounts of cobalt-57, cobalt-60, and cesium-137 provided by Japan Radioisotope Association. The dependence of the detection efficiency on the height of the sample in the container was also determined using the same standard materials. We measured the reference soil sample of IAEA-375 to validate our calibration before sample measurement.

Leaching of cesium and iodine into water at various $\mathrm{pH}$ was conducted by adding $15 \mathrm{~mL}$ of water into $5.0 \mathrm{~g}$ of surface soil sample from Site 3 with subsequent $\mathrm{pH}$ adjustment with small aliquots of $\mathrm{NaOH}$ or $\mathrm{HCl}$ solution. Dissolved fractions of iodine and cesium were determined by $\gamma$-ray measurement as described above. To determine the iodine fraction incorporated into humic substances, two-step experiments were conducted (Yamada et al., 1999): (i) a solution leached with $0.10 \mathrm{M} \mathrm{NaOH}$ was acidi- 
fied to $\mathrm{pH} 2$ to precipitate humic acid; (ii) the fulvic acid fraction was determined by its removal from the filtrate of procedure (i) through the coprecipitation of fulvic acid into calcium oxalate. In these procedures, the radioactivities of iodine and cesium were determined by employing the method described above.

\section{RESULTS AND DISCUSSION}

Vertical profiles at the three sites are shown in Fig. 1. The radioactivity of iodine-131 was corrected to the value for April 13 (the sampling date) based on its half life. By summing the radioactivity over the whole depth, inventories can be determined considering the area of the soil core. The inventories of iodine-131 were 49.0-66.3, 10.4, and $94.9 \mathrm{kBq} / \mathrm{m}^{2}$ at Sites 1-3, respectively. Those for cesium-137 were $313-334,26.9$, and $129 \mathrm{kBq} / \mathrm{m}^{2}$, respectively. All of iodine-131 originating from global fallout already decayed. The cesium-137 inventories were far higher than those of the global fallout of cesium-137 in Japan $\left(c a .2-5 \mathrm{kBq} / \mathrm{m}^{2}\right)$ from atmospheric nuclear tests in the 1960s (Yamamoto et al., 1983; Sakaguchi et al., 2009, 2010). Consequently, the results show that the radionuclides did not originate from global fallout, but were emitted from the Fukushima Daiichi Nuclear Power Station during the 2011 accident.

The vertical profiles in Fig. 1 show that iodine and cesium were distributed close to the surface. Proportions of contents at depths of $0-5 \mathrm{~cm}$ to total amounts were more than $87 \%$ for cesium-137 at the three sites, demonstrating that cesium did not migrate into deeper layers even 1 month after the accident and possibly the deposition on the soil (Table 1). The proportion of iodine-131 in the $0-5 \mathrm{~cm}$ layer was more than $76 \%$, also showing its high affinity for soil component. However, the proportions of iodine at depths of $0-2.5$ and $0-5 \mathrm{~cm}$ were smaller than those of cesium (except at Site 2, where almost all cesium and iodine were retained within $2.5 \mathrm{~cm}$ depth), suggesting that iodine is more mobile than cesium in the soil.

Water leaching experiments showed that iodine is much more extractable than cesium into the aqueous phase at various $\mathrm{pH}$, especially under an alkaline condition (Fig. 2 ). Less than $1 \%$ of cesium is dissolved into the aqueous phase at any $\mathrm{pH}$. The soil sample was also washed with $2.0 \mathrm{M} \mathrm{HCl}$, but still less than $1 \%$ of cesium was found in the $\mathrm{HCl}$ solution. These results are consistent with the fact that iodine is distributed to a deeper layer to a larger degree than cesium (Table 1 ). The very high affinity of cesium to soil indicated here can be explained by the strong interaction of cesium with clay minerals (Bostick et al., 2002; Tsukada et al., 2008).

Acidification and calcium oxalate experiments showed that $c a .40 \%$ of iodine initially leached into $0.10 \mathrm{M} \mathrm{NaOH}$ solution was incorporated into humic and fulvic acids. The formation of organo-iodine in soil has recently been suggested in several studies, especially under an oxic condition as in the surface soil (Yamaguchi et al., 2010; Shimamoto et al., 2011). Because it has been suggested that iodine may be supplied mainly as inorganic forms in rainwater as was found in the Chernobyl accident (Muramatsu et al., 1987), our results showed that the formation of organic iodine must have occurred within 1 month in the soil. Considering the facts that (i) iodine can dissociate from humic substances under an alkaline condition (Shimamoto et al., 2011) and (ii) extraction by $0.10 \mathrm{M} \mathrm{NaOH}$ cannot dissolve the humin fraction (i.e., the main component of humic substances) (Stevenson, 1994), the present results suggest that iodine is mainly in organic form.

\section{SUMMARY}

This study suggests that the migration of iodine-131 and cesium-137 into a deeper soil layer (deeper than 5 $\mathrm{cm}$ ) is restricted due to their strong affinities for humic substances and clay minerals, respectively. The fact that the radionuclides were retained at the very surface is important information for the decontamination of the radionuclides from soil in future remediation.

\section{REFERENCES}

Bostick, B. C., Vairavamurthy, M. A., Karthikeyan, K. G. and Chorover, J. (2002) Cesium adsorption on clay minerals: An EXAFS spectroscopic investigation. Environ. Sci. Technol. 36, 2670-2676.

Butler, D. (2011) Radioactivity spreads in Japan. Nature 471, 555-556.

Imanaka, T., Endo, S., Sugai, M. and Ozawa, S. (2011) Report for the radiological survey in the Iidate-mura settlement: Effect of Fukushima nuclear power station accident. Kagaku 81, 594-600, Iwanami (in Japanese).

Muramatsu, Y., Sumiya, M. and Ohmomo, Y. (1987) Iodine131 and other radionuclides in environmental samples collected from Ibaraki/Japan after the Chernobyl accident. Sci. Total Environ., 67, 149-158.

Sakaguchi, A., Kawai, K., Steier, P., Quinto, F., Mino, M., Tomita, J., Hoshi, M., Whitehead, N. and Yamamoto, M. (2009) First results on ${ }^{236}$ U levels in global fallout. Sci. Total Environ. 407, 4238-4242.

Sakaguchi, A., Kawai, K., Steier, P., Imanaka, T., Hoshi, M., Endo, S., Zhumadilov, K. and Yamamoto, M. (2010) Feasibility of using ${ }^{236} \mathrm{U}$ to reconstruct close-in fallout deposition from the Hiroshima Atomic Bomb. Sci. Total Environ. 408, 5392-5398.

Shimamoto, Y. S., Takahashi, Y. and Terada, Y. (2011) Formation of organic iodine supplied as iodide in a soil-water system in Chiba, Japan. Environ. Sci. Technol., 45, 2086-2092. Stevenson, F. J. (1994) Humus Chemistry: Genesis, Composi- 
tion, Reactions. 2nd ed., 24-58, Wiley.

Tsukada, H., Takeda, A., Hisamatsu, S. and Inaba, J. (2008) Concentration and specific activity of fallout ${ }^{137} \mathrm{Cs}$ in extracted and particle-size fractions of cultivated soils. $J$. Environ. Radioact. 99, 875-881.

Yamada, H., Kiriyama, T., Onagawa, Y., Hisamori, I., Miyazaki, C. and Yonebayashi, K. (1999) Speciation of Iodine in Soils. Soil Sci. Plant Nutr. 45, 563-568.
Yamaguchi, N., Nakano, M., Takamatsu, R. and Tanida, H. (2010) Inorganic iodine incorporation into soil organic matter: evidence from iodine K-edge X-ray absorption nearedge structure. J. Environ. Radioact. 101, 451-457.

Yamamoto, M., Komura, K. and Sakanoue, M., (1983) ${ }^{241}$ Am and Plutonium in Japanese rice-field surface soils. J. Radiat. Res. 24, 237-249. 